\title{
Evaluation of the Arabic version of the Beck scale
}

\author{
Khalid Mohammed Almahrag (Corresponding author) \\ Special Education, King Saud University \\ 3749 Riyadh 12372 - 7959, Kingdom of Saudi Arabia \\ E-mail: almahrej@yahoo.com
}

\begin{abstract}
The main objective of this paper is to provide a general vision of codifying the Beck measure of despair on a representative sample of Arab societies in terms of their suitability for Arab culture. Many of these studies have investigated the metric characteristics of a scale of despair on cultural, economic, and social examples from different Arab societies. This difference may impact the realism, credibility, and measurement of Beck's distress to measure the purpose prepared to measure it.
\end{abstract}

Keywords: Beck scale, Arabic scale, Evaluation scale

DOI: $10.7176 /$ RHSS/11-18-08

Publication date:September $30^{\text {th }} 2021$

\section{Introduction}

Some negative psychological phenomena have begun to spread in the Arab countries, including the phenomenon of suicide in recent times for several cases that the media occasionally mention. We are not aware of some other sensations, such as isolation and social withdrawal caused by political and ideological changes in the Arab world, such as the Arab Spring and the recent economic crisis in which the world has suffered. As a result of the Krona pandemic, this will undoubtedly lead these communities to psychological and social problems that have individual research that is in different forms, including despair and suicide in the most potent form. As a result, there must be predictive measures that give a perception of the situation of community members, such as the Beck measure of despair.

Beck\& Steer (1974) set out to prepare the Beck Hopelessness Scale from a desperate view that "a sad emotional state characterized by the individual's negative expectations for life and the future (Beck et al.,1985) symbolizes this BHS scale of 20 pandas developed for each item. Of these two possible answer items (yes or no), this measure measures three manifestations (feeling towards the future, loss of motivation, negative expectations). It performs the task of predicting suicidal behavior and has been codified into several images in several different cultures such as Brazilian, Japanese, Chinese, Portuguese, German, Australian, Swedish, Canadian, and French, and some Arab communities such as Syria, UAE, Kuwait, and Saudi Arabia.

\section{BHS's Measure of Despair}

The Beck scale is a suitable measure for use due to its small size, ease of use and correction, and the indications of Validity and reliability achieved on the original image scale. The strength of the Beck Despair Scale "original image" is calculated in several different ways. For example, internal consistency using the Cronbach Alpha equation and the alpha factor for a sample of 1,172 depressed people estimate to be 0.90 (Steer et al.,1993). It also calculated a sample alpha coefficient of 120 depressed elderly to 0.85 (Hill et al.,1988). The scale's reliability is also calculated in another way, like the replay where the first and second application was separated for three weeks, reaching the reliability factor for a sample of 149 university students to 0.78 (Holdenand\&Feekkan,1998).

Some studies conducted to verify the ability of the Beck Discriminatory Despair Scale indicate a high degree of discriminatory Validity of the scale. The scale applied to a sample of psychopaths matched by a selection of psychiatric patients, which found an indication among the average scores of the two groups at a function rate of 0.01 (Street et al.,1993). He also studied the converging and divergent Validity of the scale by calculating its correlation with several other measures that measure the characteristics of its affinity for beck's action of despair, such as measures of depression, anxiety. Moreover, neuroses, such as optimism, social acceptance, and timetaking, and the results obtained within the limits of convergence and divergence are expected (Sharon \& Oliver, 1985). studies showed positive correlations of convergence measures, with a Beck scale of 0.46 to 0.67 and negative correlations with the scales of its distances of 0.43 to -0.68 . Some studies were conducted on the Beck scale to verify the charity of the workers. Including the 1974 study of Beck and his colleagues. The survey of 
Spruce and his colleagues in 1996 demonstrated. The working Validity of the scale. These studies on the sincerity and reliability of the scale indicated. The integrity of the scale to measure the characteristic to be measured. Therefore, the Beck scale is a good measure of despair, so we chose this measure to study it.

There is no doubt that the Arab countries suffer from a severe lack of psychological measures of all kinds, especially subjective measures such as depression, despair, anxiety. And pessimism. The political, economic, social, and health changes that have prevailed in the world in recent times predicted the emergence of psychological differences among members of society. Requires addressing such changes by identifying them and trying to anticipate and address them. So we are interested in using some psychological measures for the character, which enables us to remember the psychological dangers facing us, such as suicide. May result from the feeling of despair of the individual. Moreover, the Beck measure of pain is one of the measures considered in this regard, codified in several languages and cultures. Its characteristics have shown a metric of confidence, which invites us to benefit from, by codifying it on different samples such as Arab societies.

\section{Rationing beck's measure of despair on Arab societies}

Before starting to legalize the Beck scale on Arab societies, several necessary steps must be taken into account, including taking the approval of officials in the relevant ministries such as health and education, where it includes studying the legalization of the scale on samples and arbitrators from those ministries or their followers, and the task force of rationing the scale can consist of a specialist in measurement whose task is to study its sincerity and reliability and verify the safety of its items and specialists in English is a translation and transfer mission from English to Arabic, a statistician who extracts and analyzes statistical evidence, measurement results, a psychologist who studies the characteristic for which the scale is to be designed, and an economist who determines the overall budget of the project by accurately determining the project's revenues and expenses, a data entry and a secretary, and since the scale is relatively small, I think that It takes up to three months to be legalized as an approximate period that can be increased and decreased.

The first step to codifying your scale on the Arab environment is to translate the 20 items of the scale by translating them separately by three specialists in each English language without informing anyone of the translation of the other and then the lead researcher compares those translations to confirm the phrases that have been reached to agree on their translation and exclude the paragraphs that have been different in translating them for re-examination, where they may be the phrases that have been different in their translation from their lack of clarity to express the original meaning of these items in the original scale and the ambiguity of they need to be re-transferred with him and then presented again for final translation to the number of three faculty members of a university specializing in English for arbitration and take their notes on the scale items for review, and then send the picture For an Arabic scale extracted to an English specialist to translate it backwards from Arabic to English until it is confirmed that it has returned to its original image, if it returns to the original image, this is an important indicator that proves that the metric phrases are clearly transmitted. To ensure that the measured words are sent from English to Arabic. We must test it on several graduate students in the English department so that students are exposed to answer the Arabic image of the scale. They were followed by directly telling them to the original English image to ascertain the virtual Validity of the scale and then come up with the final appearance of the scale.

Several methods can verify the reliability of the Arabic image of the Beck scale, such as the internal consistency method and using the half-half method with correction with the Spearman-Brown equation. As well as the replay method can use to calculate the scale's reliability so that the scale is applied in Its Arabic form to a group of testers and then returned after a certain period not exceeding three weeks on the same sample of the testers.

The Validity of the Arabic image can be studied from the scale in several ways, including by checking discriminatory Validity by applying it to a sample of graduate students and the second sample of psychiatric patients at the same age. What is required here? It is to identify the degree of difference between grades of the testers that we can see the Validity of what the scale predicts as the scale expects to show a differentiation between the grades of the testers in both categories of the study sample (worse and worse psychiatric patients).

Moreover, we can study the convergence and divergence Validity of the proposed Arabic image. For the scale by comparing its results with several measurement results for its convergence of the characteristic measured by the proposed measure of insulation (Beck's measure of despair), such as depression and anxiety measures and measures of its divergence of factors measured by the proposed measure of rationings (Beck's measure of pain), such as the measures of simplicity or happiness. This test conduct to validate. The scale on the same sample in application on all efforts to note. The convergence of results with the criteria for its convergence and divergence 
with the standards of its distance. For example, we expect the results to converge. Means of anxiety, keeping away from stress, and keeping them away from the measures - results with a scale of simplicity.

\section{Conclusion}

There is no doubt that this paper provided a clear vision of what to do to codify your scale on the Arab environment based on several considerations. The most important of which is that many of the legalization procedures for your measure of despair are carried out on different Arab backgrounds. The researcher calls for legalization on the scale, considering the diversity of rationing samples from Arabic speakers.

\section{References}

Beck, A.T., Steer, R.A., Kovacs, M., \& Garrison, B. (1985) Hopelessness and eventual suicide: a 10- year prospective study of patients hospitalized with suicidal ideation. American Journal of Psychiatry, 142,559-563.

Steer, R.A., Kumar, g., \& Beck, A.T. (1993). Hopelessness in adolescent psychiatric inpatients. Psychological Reports, 72,559-564.

Hill, R.D., Gallagher, D., Thompson, L.W., \& Ishida, T. (1988). Hopelessness as a measure of suicidal intent in the depressed elderly. Psychology and Aging, 3,230-232.

Holden, R.R., \& Fekken , G.C.(1998). Test-retest reliability of the Hopelessness Scale and its items in a university population. Journal of Clinical Psychology: 43,597-600.

Sharon, L, L \& Oliver, J. M (1985). the Beck Inventory: Psychometric properties in University Students. Journal of Personality Assessment,49,4,434-436. 